\title{
Content Based Thermal Images Retrieval
}

\author{
Hernán Darío Benítez and Gloria Inés Alvarez \\ Pontificia Universidad Javeriana Cali, \\ Calle 18 No. 118-250, Cali, Colombia \\ \{hbenitez, galvarez\}@javerianacali.edu.co \\ http://www.javerianacali.edu.co
}

\begin{abstract}
INDT (Infrared Nondestructive Testing) inspections produce large sets of thermal images. The thermal images obtained must be stored to do comparisons with the results of previous and future inspections generating considerable amounts of data to be analyzed. In this paper, we present a CBIR system based on thermal features and spatial relations among defects in the thermal image to measure the similarity between query and database images affected by non uniform heating and taken from anisotropic material samples.
\end{abstract}

Keywords: Content based image retrieval, spatial relationships, infrared nondestructive testing, thermal contrast.

\section{Introduction}

Infrared Nonsdestructive Testing (INDT) is a nondestructive evaluation technique in which the specimen surface is thermally stimulated to produce a temperature difference between sound (free of defects) areas and eventual defective regions. This technique is fast, non-invasive and is used in several industry fields such as automotive and aircraft industry [1]. In aircfrat industry, INDT is well suited for in-service damage detection in CFRP (Carbon Fiber Reinforced Plastic) 2 structures since it is well suited to detect shallow delaminations and can be deployed directly on site.

INDT inspections produce large repositories of digital thermal images. The thermal images obtained must be stored to do comparisons with the results of previous and future inspections, generating considerable amounts of data to be analyzed. Effective extraction of visual and temperature features is needed to provide meaningful access to visual data and retrieve thermal images based not on textual annotations but on visual features [3]. We propose the application of content-based image retrieval (CBIR) for thermal images obtained from INDT inspections. CBIR allows the retrieval of relevant images based on a pre-defined similarity measure between image features. For example, this type of system could be used to measure the true temperature on Printed Circuit Boards (PCB) that are produced in a large scale by the electronic industry. In this paper we present the thermal image representation based on thermal constrast and spatial relations between defects found in the image. Then, we describe the image

A. Elmoataz et al. (Eds.): ICISP 2010, LNCS 6134, pp. 479-487, 2010.

(C) Springer-Verlag Berlin Heidelberg 2010 
retrieval process and discuss about the results obtained and finally we present the conclusions and further work.

\section{Thermal Image Representation}

Temperature information allows to isolate hot spots and the absence of clear shape information makes hard the object recognition task since important image features (defects) appear as smooth transitions in the temperature images due to the thermal diffusion process. To take advantage of these special features we use two sources of information for representation: thermal contrast and spatial relations. Thermal contrast enhances defect visibility and spatial relations give an idea about the relative position of each defect in the image.

The hypothesis is that these sources of information capture similarity between thermal images since the existence of hot spots (defects inside the material) and their relative position in the thermal image are relevant to human similarity perception. Our images are obtained from simulation and correspond to CFRP (Carbon Fiber Reinforced Plastic) samples with air defects that simulates delaminations (i.e layer separations filled with air) of variable size and depth. In the next section we present the image segmentation and feature extraction followed to represent thermal images.

\subsection{Segmentation}

In several situations when INDT is used, the important point is whether or not a defect is present in a given part of the sample 4]. The segmetation algorithm makes use of some heuristics and is based on the fact that INDT images have a limited number of spatial features. The first step is to localise the defect and then specific thresholds are found in the image to estimate the border of the defects. Each threshold is found by means of a region growing approach, which starts at the central point of a defect and stops when either an image border is hit or the number of pixels agglomerated together around the seed (central point) increases abruptly. The segmentation algorithm makes use of the constant MDN (Minimum Neighbour Distance) established by trial and error. Since MND represents a distance between pixels rather than an absolute value, it is independent of the image but depends on the field of view size and on the minimum defect size necessary for detection.

\subsection{Features Extraction and Defects Representation}

Segmentation output is regions of pixels that belong to defects. In the feature extraction stage, the resulted set of segmented pixels is converted in a form suitable for computer processing [5]. Defects are represented as complete region since we are interested in an internal property: thermal contrast. It is used in INDT to evaluate defect visibility and enhance image quality. Several types of 
contrasts have been defined such as absolute contrast, relative contrast, normalized contrast and standard contrast [6]. All these contrast definitions require the use of the temperature in a sound area whose definition is a critical issue. In a wide sense, its locations are not precisely identified since it may not be known in advance where the defects are, if present at all [7. The modified Differential Absolute Contrast (m-DAC) was developed to perform a more convenient computation of the sound area temperature by using the thermal quadrupoles theory 8 , 9]. The thermal quadrupoles method is a Laplace transform based technique to explicitly represent linear systems and it is used to solve heat transfer problems. This method transforms the differential equations from the time-space domain representation to a Laplace domain representation that exhibits a very simple explicit form. Absolute thermal contrast is calculated as $\Delta T=T_{d}-T_{n d}$ where $T_{d}$ is temperature on defective area and $T_{n d}$ is temperature on nondefective area. The relative contrast is $\frac{\Delta T}{T_{n d}}$

For absolute m-DAC the $T_{n d}$ is calculated as :

$$
T_{n d}=\frac{\left.L^{-1}\left[\frac{\operatorname{coth} \sqrt{\frac{p L^{2}}{\alpha}}}{\sqrt{p}}\right]\right|_{t}}{\left.L^{-1}\left[\frac{\operatorname{coth} \sqrt{\frac{p L^{2}}{\alpha}}}{\sqrt{p}}\right]\right|_{t^{\prime}}} T_{d e f}\left(t^{\prime}\right)
$$

and the relative $\mathrm{m}-\mathrm{DAC}$ is:

$$
\Delta T_{m-D A C r e l}=\frac{T_{d e f}(t)}{T_{n d}(t)}-1
$$

where $T_{\text {def }}(t)$ is the temperature on a defective or sound area, $L$ is the specimen thickness, $p$ is the Laplace variable, $\alpha$ is the thermal diffusivity and $t^{\prime}$ is the instant of time at which the first defect in the sample becomes visible.

This thermal contrast definition is used to represent the defects given its ruggedness to uneven heating that is commonly presented in INDT experiments. Each defect is represented by the mean $\mu$ and standard deviation $\sigma$ of a segmented region found in the maximum contrast image. On the other hand, we define the standardized Euclidean distance $D_{a}$ to measure the disimilarity between entities $A$ and $B$ (defects) represented by thermal contrast features:

$$
D_{a}(A, B)=\sqrt{\sum_{i=1}^{p}\left(\frac{A_{i}-B_{i}}{\sigma_{i}}\right)^{2}}
$$

where $\sigma_{i}$ is the standard deviation of the $i$-th dimension over the number of defects found in the thermal image and $p$ the length of the feature vector, which, in this case is two.

\subsection{Spatial Relations Representation}

The representation of spatial relations proposed for each spatial relation is called Weighted Walkthrough [10, [1]. This representation is tolerant to translations 
and rotations, it includes a distance metric, and its implementation is efficient in space and affordable in time.

Given two entities $A$ and $B$ from one image, each one represented by a cloud of dots, spatial relation from A to $\mathrm{B}$ is expressed by a matrix $M$ of size $3 \times 3$, which counts in each position the number of dots of both entities with a given spatial relationship between them. Notice that this matrix is not commutative, that is, it represents the relation from entity $A \operatorname{dot}\left(x_{A}, y_{A}\right)$ to entity $B$ dot $\left(x_{B}, y_{B}\right)$. This representation covers all the possible relations from one dot to another: up $(1,2)$, low $(3,2)$, left $(2,1)$, right $(2,3)$, upper left $(1,1)$, upper right $(1,3)$, lower left $(3,1)$, lower right $(3,3)$ and overlapped $(2,2)$.

To calculate the distance between two spatial relationships $w$ and $w^{\prime}$ (each one is a matrix of $3 \times 3$ size). The distance suggested by Berreti et. al [1] is:

$$
\begin{aligned}
D_{s}\left(w, w^{\prime}\right)= & \lambda_{H} d_{H}\left(w, w^{\prime}\right)+\lambda_{V} d_{V}\left(w, w^{\prime}\right)+\lambda_{D} d_{D}\left(w, w^{\prime}\right)+ \\
& \lambda_{H_{0}} d_{H_{0}}\left(w, w^{\prime}\right)+\lambda_{V_{0}} d_{V_{0}}\left(w, w^{\prime}\right)+\lambda_{00} d_{00}\left(w, w^{\prime}\right)
\end{aligned}
$$

Where all $\lambda$ coefficients are nonnegative numbers with their sum equal to one. And terms $d_{H}, d_{V}, d_{D}, d_{H_{0}}, d_{V_{0}}$ y $d_{00}$ are calculated as follows:

$$
\begin{aligned}
d_{H}\left(w, w^{\prime}\right) & =\left|\left(w_{1,3}+w_{3,3}\right)-\left(w_{1,3}^{\prime}+w_{3,3}^{\prime}\right)\right| \\
d_{V}\left(w, w^{\prime}\right) & =\left|\left(w_{1,1}+w_{1,3}\right)-\left(w_{1,1}^{\prime}+w_{1,3}^{\prime}\right)\right| \\
d_{D}\left(w, w^{\prime}\right) & =\left|\left(w_{3,1}+w_{1,3}\right)-\left(w_{3,1}^{\prime}+w_{1,3}^{\prime}\right)\right| \\
d_{V_{0}}\left(w, w^{\prime}\right) & =\left|\left(w_{2,1}+w_{2,3}\right)-\left(w_{2,1}^{\prime}+w_{2,3}^{\prime}\right)\right| \\
d_{H_{0}}\left(w, w^{\prime}\right) & =\left|\left(w_{3,2}+w_{1,2}\right)-\left(w_{3,2}^{\prime}+w_{1,2}^{\prime}\right)\right| \\
d_{00}\left(w, w^{\prime}\right) & =\left|\left(w_{2,2}-w_{2,2}^{\prime}\right)\right|
\end{aligned}
$$

Image representation includes both entities and spatial relationship representations. Joint distance $\mu$ between images $Q$ and $D$ (query and database image), given an interpretation $\Gamma$ 1 , can be established as follows:

$$
\mu^{\Gamma}(Q, D)=\lambda \mu_{a}^{\Gamma}(Q, D)+(1-\lambda) \mu_{s}^{\Gamma}(Q, D)
$$

Where $\mu_{a}$ and $\mu_{s}$ are attribute and spatial distances.

$$
\begin{aligned}
& \mu_{a}^{\Gamma}(Q, D)=\sum_{k=1}^{N_{q}} D_{a}\left(q_{k}, \Gamma\left(q_{k}\right)\right) \\
& \mu_{s}^{\Gamma}(Q, D)=\sum_{k=1}^{N_{q}} \sum_{h=1}^{k-1} D_{s}\left(\left[q_{k}, q_{h}\right],\left[\Gamma\left(q_{k}\right), \Gamma\left(q_{h}\right)\right]\right)
\end{aligned}
$$

In Equation (5),$\lambda$ is a nonnegative number in the range $[0,1]$, distance $D_{a}$ is defined in section 2.2 and distance $D_{s}$ is defined in Equation (44). In Equation (6) $N_{q}$ is the number of entities in image $Q$.

\footnotetext{
${ }^{1}$ An interpretation is a matching among entities of both images. If $a$ is an entity of image $Q, \Gamma(a)$ is an entity of image $D$ that corresponds to $a$.
} 


\section{$3 \quad$ Image Retrieval}

In an image retrieval system each image into the database must be represented by a graph, that is, a pair $(E, R)$ where $E$ is a list of entity representations and $R$ is a list of spatial relation representations between entities. When a query image is presented to the system, it calculates the representation for such new image, a pair $\left(E^{\prime}, R^{\prime}\right)$, and proceeds to compare this image against database ones obtaining their distances. The lower the distance, the higher the similarity. Algorithm 1 details the retrieval process, its inputs are: a query image represented as a matrix of pixels $(\mathrm{Q})$, a data base of images previously represented as a graph of entities and spatial relations (DB), and an integer value $(k)$ used to select images for the answer set: the first $k$ images of the database with the shorter distances with respect to the query image will be part of the answer set. Once the algorithm begins, first step consist of processing the query image to get its representation (line 4), that is, its entities and spatial relations. Then, we iterate on database to compare each image against query one (line 5), for each image from database, we perform several actions: in line 6 we calculate interpretation $\Gamma$ which is a relationship among entities of both images, that is, we choose for each entity of the query image the entity of the data base image with the shortest distance to it. If the number of entities in both images is different, some entities will not be matched causing a penalty in the distances computation. This matching between entities of both images is the core of the retrieval process because it is necessary in the following steps. In lines 7, 8 and 9 distances among entities, spatial relations and images are calculated depending on the interpretation $\Gamma$ and following the method described in Section 2, Once we have calculated all the distances, we sort them in increasing order (line 12) and report the $k$ first images in the ranking (lines 13-18).

\section{Results and Discussion}

The CBIR system was tested on 36 CFRP samples with air delaminations simulated with ThermoCalc 6L [12] a software intended for calculating 3D (threedimensional) temperature distributions in anisotropic solids that may contain subsurface defects. The corresponding mathematical heat conduction problem is modeled in Cartesian coordinates and solved by using an implicit finitedifference numerical scheme. The simulated samples were affected by a phenomena easily found in INDT experiments named non-uniform heating, which is the non-homogeneous heat distribution on the specimen surface. The image set is made of four subsets of nine images. Each subset is generated from position and depth variations of defect spatial (air gaps between layers) distributions shown in Figure 1]

Each image in every subset is considered as a query image varying the dimension of the retrieval set from 1 to 27 as shown in Figures 2 and 3 where the retrieval performance is obtained by increasing an accumulator in one when the image retrieved belongs to the same query image subset and normalizing by 


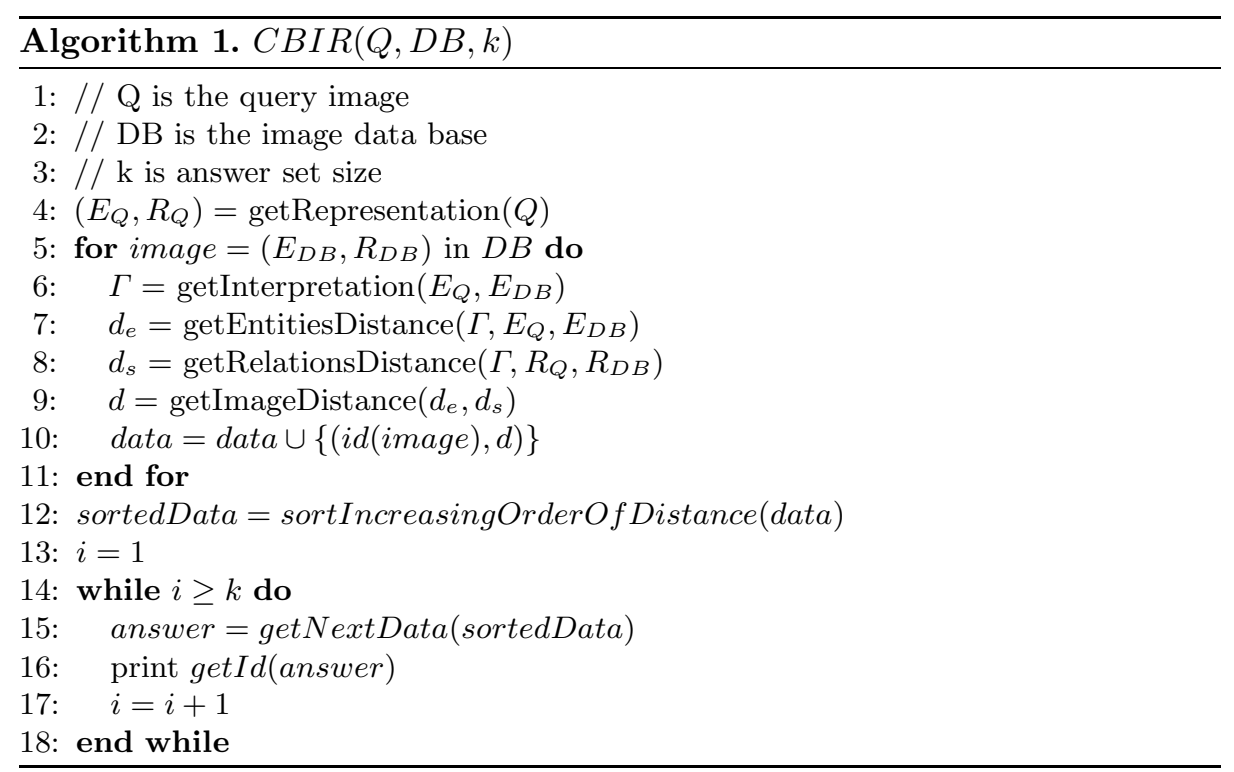

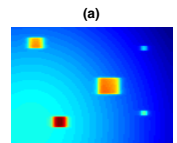

(c)

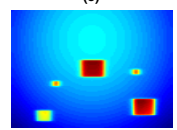

(b)

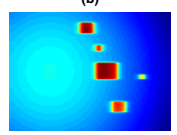

(d)

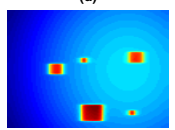

Fig. 1. Defect spatial distribution for subsets: (a) I, (b) II, (c) III and (d) IV

the subset size (9). In Figure 2(a) for the nine query cases $80 \%$ of performance is reached for a retrieval set dimension of 14 . On the other hand, for the nine query cases represented in Figure 2(b), a $100 \%$ performance is reached for the same retrieval dimension. In Figure 3(a) is shown that only for queries 1 and 2 the $100 \%$ of performance was reached and for the other queries the maximum performance reached was roughly $80 \%$ since thermal images 1 and 2 were not retrieved even in the largest retrieval set dimension. Figure 3.b) shows that 100 $\%$ of performance was obtained with a retrieval set dimension of 14 . According to these results the CBIR system is able to retrieve similar thermal images to a query image even when these are affected by non-uniform heating and the material is anisotropic (i.e different thermal diffusivity for each spatial direction) as in the case of CFRP.

Precision vs. recall graphs for all the subsets are in Figure 4 and 5, they present the behavior of 4 query images from each subset. In subset I, the best balance precision-recall is reached near to 0.8 for recall and near 0.7 for precision, as can be 


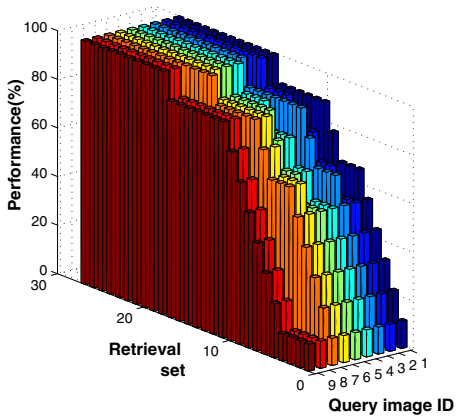

(a)

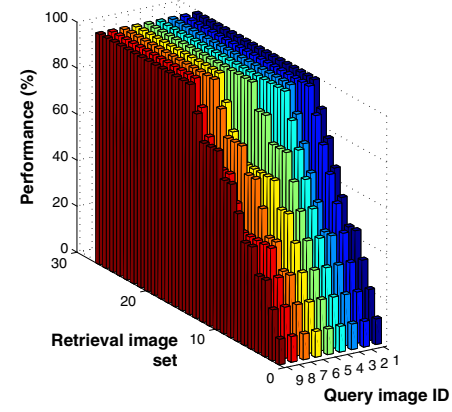

(b)

Fig. 2. Performance results for subsets (a)I and (b) II

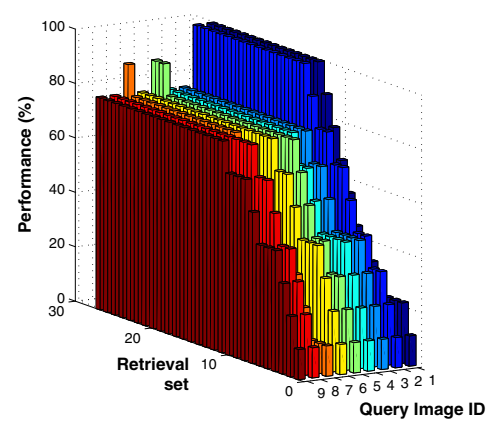

(a)

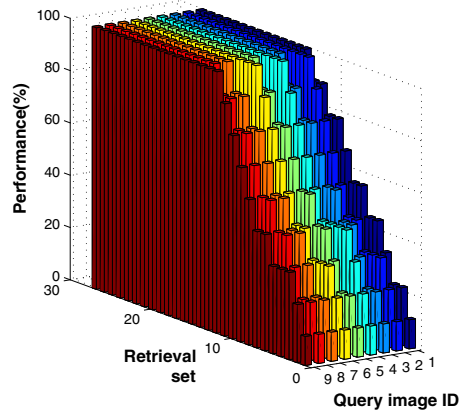

(b)

Fig. 3. Performance results for subsets (a)I and (b) II

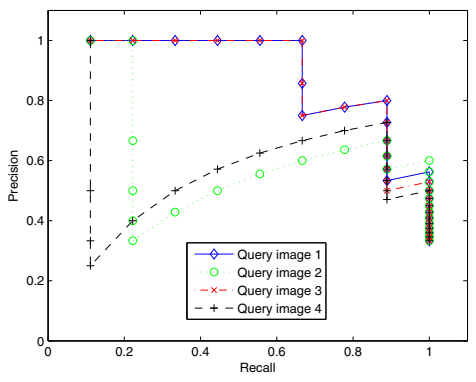

(a)

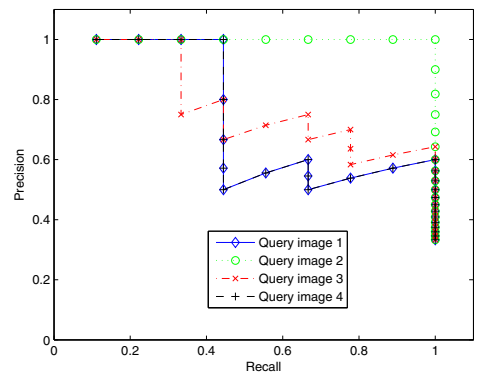

(b)

Fig. 4. Precision vs Recall for subsets (a) I and (b) II considering retrieval set sizes from 1 to 27 


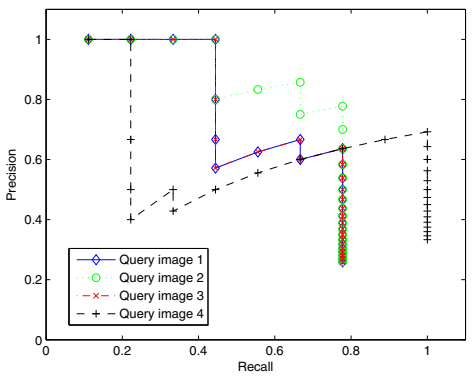

(a)

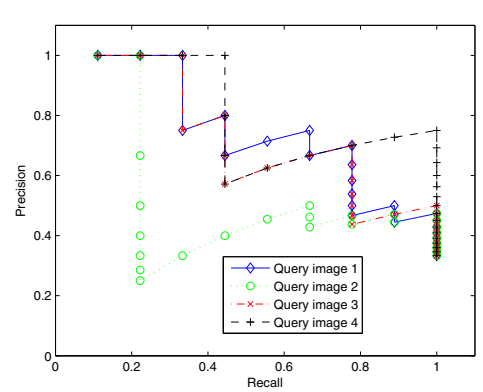

(b)

Fig. 5. Precision vs Recall for subsets (a) III and (b) IV considering retrieval set sizes from 1 to 27

seen in Figures 4(a); in subset II (Figure 4(b), we get a successful retrieval process with 1.0 both in precision and recall and other results with recall 1.0 and precision around 0.6 ; in subset III (Figure 5(a)) best values are around 0.6 in precision and recall; in subset IV (Figure $5(\mathrm{~b})$ ), best balance is reached in precision value of 0.75 and recall of 1.0. In all subsets, these best values are reached with retrieval sets sizes between 8 and 12. In sets I, II and IV all the tests obtained recall equal to 1.0 after the best balance point, meaning that our method is prone to include all the relevant answers in the set although it implies sometimes the inclusion of some false positive results too, for this reason precision is lower.

\section{Conclusions}

In our paper we proposed a CBIR system for thermal image retrieval. The query is image content based. The relative modified DAC thermal contrast is used to represent defects obtained by a semiautomatic thermal image segmentation stage based on region growing approach. This representation is specially useful when thermal images are affected by non uniform heating since it depends less on the absorbed energy and is less affected by surface optical properties. Besides thermal information the spatial relations among defects was taken into account to measure the similarity between database and query images. This combined approach (thermal contrast plus spatial information) allows to build a CBIR system which retrieves thermal images affected by non uniform heating from anisotropic material specimens. However, if other material needs to be inspected, the representation of each image must be obtained again as well as the spatial relations. Finally, the system will be tested with images obtained from experimental data and electrical equipment inspection.

\section{References}

1. Maldague, X.: Theory and Practice of Infrared Technology for Nondestructive Testing. Wiley Interscience, New York (2001)

2. Guizzo, E.: Carbon takeoff. IEEE Spectrum 43(1), 24-28 (2006) 
3. Perner, P.: Content-based image indexing and retrieval in an image database for technical domains. In: Horace Ip, H.S., Smuelder, A. (eds.) Multimedia Information Analysis and Retrieval. LNCS, vol. 1464, pp. 207-223. Springer, Heidelberg (1998)

4. Maldague, X., Krapez, J.C., Poussart, J.C.: Thermographic nondestructive evaluation (nde): an algorithm for automatic defect extraction in infrared images. IEEE Transactions on Systems, Man and Cybernetics 20, 722-725 (1990)

5. Gonzalez, R., Woods, R.: Digital image processing. Prentice-Hall, Englewood Cliffs (2001)

6. Bendada, A., Maillet, D., Batsale, J.C., Degiovanni, A.: Reconstitution of a non uniform interface thermal resistance by inverse heat conduction application to infrared thermography testing. Inverse Problems in Engineering 6, 79-123 (1998)

7. Benítez, H.D., Ibarra-Castanedo, C., Bendada, A., Maldague, X., Loaiza, H., Caicedo, E.: Modified differential absolute contrast using thermal quadrupoles for the nondestructive testing of finite thickness specimens by infrared thermography. In: CCECE 2006 - Canadian Conference on Electrical and Computer Engineering, Paper No. 398, Ottawa (Ontario) Canada, Mayo 7-10 (2006)

8. Benítez, H.D., Ibarra-Castanedo, C., Bendada, A., Maldague, X., Loaiza, H., Caicedo, E.: Definition of a new thermal contrast and pulse correction for defect quantification in pulsed thermography. Infrared Physics \& Technology 51(3), 160-167 (2008)

9. Maillet, D., André, S., Batsale, J., Degiovanni, A., Moyne, C.: Thermal quadrupoles: solving the heat equation through integral transforms. John Wiley and Sons, West Sussex (2000)

10. Berreti, S., del Bimbo, A., Vicario, E.: Modelling spatial relationships between colour clusters. Pattern Analysis \& Applications 4, 83-92 (2001)

11. Berretti, S., Del Bimbo, A., Vicario, E.: Weighted walkthroughs between extended entities for retrieval by spatial arrangement. IEEE Transactions on Multimedia 5(1), 52-70 (2003)

12. Vavilov, V.: Three dimensional analysis of transient thermal ndt problems by data simulation and processing. In: Thermosense XXII Proceedings SPIE, vol. 4020 (2000) 